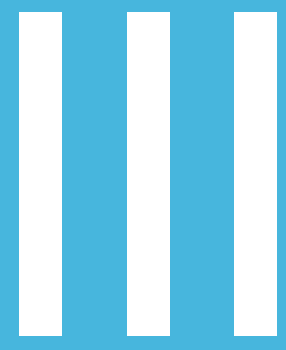

\title{
LOS PROVERBIOS BÍBLICOS EN EL DIÁLOGO COMO MÉTODO PARA LA SOLUCIÓN DE PROBLEMAS
}

\section{Bible Proverbs on dialogue as a method of problem solving}

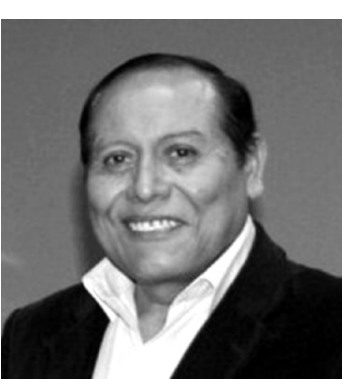

\section{Raúl Acuña Casas}

Licenciado en Matemáticas por la Universidad Nacional Mayor de San Marcos. Magíster en Educación por la Universidad Peruana Unión. Doctor en Administración por la Universidad Federico Villareal. Doctor en Psicología por la Universidad Inca Garcilaso de la Vega. Exdecano de la Facultad de Educación y Ciencias Humanas de la Universidad Peruana Unión. Actualmente se desempeña como Secretario de la Escuela de Posgrado de la Universidad Peruana Unión. Ha escrito numerosos artículos y es profesor visitante a nivel nacional e internacional. 


\section{Resumen}

Se fundamenta el diálogo como método entre el docente y el alumno, donde ambos interactúan para comprender y resolver problemas ligados a situaciones reales del entorno natural y social, en un nuevo escenario caracterizado por la investigación sistémica y el soporte de teorías transdiciplinares que ayudan a entender la complejidad de las cosas. Igualmente, se aborda la mecánica del diálogo y la estructura lógica que lo sustenta. Finalmente se plantea las implicaciones de un conjunto de proverbios bíblicos que enriquece la eficiencia del diálogo como método en la formulación de ecuaciones o relaciones que involucran todas las condiciones del problema.

Palabras clave: Diálogo, complejidad, transdisciplinariedad, lógica polirrelacional, proverbio bíblico.

\section{Abstract}

It is based on dialogue as a method between teacher and student where both interact to understand and solve problems related to real situations of natural and social environment, and in a new stage characterized by system research, and the support of Trans disciplinary theories that help us understand the complexity of things. We also touch mechanics of dialogue and logical structure that sustains. Finally, it is raised with the implications from a set of biblical proverbs that enrich the efficiency of dialogue as a method to formulate equations or relationships to involve all the conditions of the problem

Keywords: Dialogue, complexity, poly relational logic, biblical proverb, Transdisciplinarity. 
"El diálogo cómo método, es el encuentro de los hombres encauzados por conocer la verdad"

\section{Introducción}

Dado que estamos viviendo en la era del conocimiento, donde el paradigma de la complejidad se impone en la comprensión de los hechos en toda su extensión, y que un medio para alcanzar esta visión sistémica se requiere de una lógica polirrelacional que dé sentido al diálogo como método. Diálogo que permitirá adoptar una conciencia crítica no solo de nuestros puntos de vista sino del grupo en el establecimiento de las ecuaciones o relaciones entre las condiciones, datos y variables que aparecen en el problema $y$, en un contexto transdisciplinar, donde se integran el álgebra, la aritmética y la geometría. En este tipo de aprendizaje, el papel del diálogo es el entendimiento y transformación del hombre y del mundo. Así, las instituciones educativas, sean escuelas, colegios y universidades, deben operar para que el alumno angustiado por la turbulencia de la modernidad, encuentre el refugio, la ruptura de la incertidumbre por el intercambio de ideas y sentimientos con sus maestros y compañeros de clase. Se justifica que el diálogo como método traspasa toda fuente bibliográfica, porque no es con una biblioteca muerta ni como un museo, sino una relación viva que apunta a la grandeza del ser humano. Esta relación ayuda a posibilitar el desarrollo holístico del alumno.

\section{Método}

El presente artículo es producto de una investigación documental $y$, en general, en el pensamiento del autor, cuyo objetivo fundamental es innovar el diálogo a una categoría de método, para la comprensión de diferentes modelos de situaciones problemáticas, teniendo como finalidad promover la búsqueda de alternativas inventivas en la estructuración de las partes, hallazgo de la concordancia lógica, ver la pluralidad de alternativas $y$, en general, a ver el problema como un todo. Para tal efecto, siguiendo el proceso de los cuatro pasos de Polya (1990), en la solución de un problema, el diálogo se enriquece si se incorpora, en el corpus vocabular del docente, el conjunto propuesto de proverbios bíblicos. 


\section{Discusión}

\section{Presupuestos que fundamentan el diálogo como método}

Complejidad de los hechos. Se considera que los instrumentos y procesos de investigación cuantitativa, propios de las ciencias naturales, no son lo suficientemente exhaustivos en la indagación de la complejidad biológica, psicológica, sociológica y de otra ciencia humana, ya que al adoptar una de las disciplinas y dentro de esta una teoría particular, se está produciendo una observación reduccionista, lineal, unidireccional y basada en la regularidad de los hechos; suprimiendo la empírica realidad del mundo donde es posible detectar inconsistencias reales, incoherencias lógicas y hasta contradicciones conceptuales. Inclusive en las ciencias naturales, cuando se aplica el conocimiento en un contexto práctico pierde su cientificidad. Cuando una ciencia natural se manifiesta, lo hace en forma de igualdades. Sin embargo, una ecuación de igualdad es una agresión a la realidad, la reduce demasiado.

La estructura de una ciencia humana no se puede definir como un conjunto de simples entes geométricos adimensionales que no tienen color, sabor, olor, movimiento, etc., negando el valor del comportamiento como componente de cambio y de la influencia del ambiente sobre el sujeto. Un individuo no se puede considerar como si fuese un número de una cierta estructura algebraica que se sujeta a un número limitado de propiedades y reglas válidas solamente en dicha estructura y que, fuera de ella, puede perder su significatividad. En cambio, un individuo es una estructura abierta fuertemente interrelacionado con la supraestructura social, el mundo en que vive.

La previsión probabilística no solo abarca la determinación de las características sino también identificar los patrones, formas y tendencias de vida del hombre, con un alto nivel de seguridad. En tal perspectiva, un análisis basado solo en relaciones cuantitativas no agota su estudio. Las ciencias formales no pueden ser utilizadas y concebidas como parámetros adecuados de las ciencias de la vida.

La transdisciplinariedad. Busca ir "más allá", no solo de la unidisciplinariedad, sino también de la multidisciplinariedad y de la interdisciplinariedad. Su propósito es superar la parcelación y fragmentación del conocimiento que producen las distintas disciplinas particulares y su consecuente hiperespecialización. Una sola disciplina denota, su incapacidad para comprender las complejas situaciones del mundo actual, las cuales se 
caracterizan por presentar una multitud de relaciones entre los factores que la determinan.

Indudablemente, los resultados de la integración de varias disciplinas no solo serán algo más que la suma de sus partes, sino que esa sinergia también permitirá generar cuerpos de conocimientos emergentes diferentes donde los conocimientos propios de cada disciplina ya no podrán ser discernibles en ella, como tampoco podrán ser predecibles con anterioridad. Las disciplinas aisladas son menos que adecuadas para tratar los más importantes problemas intelectuales y sociales que caracterizan la vida moderna. Esa separación de saberes se torna inoperante cuando se enfrenta a la realidad concreta que vivimos: cómo disminuir los niveles de pobreza, cómo combatir eficazmente los índices de violencia y criminalidad, cómo rebajar el porcentaje del desempleo, cómo lograr una vida sana y una educación generalizadas y, en suma, cómo llegar a ofrecer una vida humana y digna a toda una población. Según von Bertalanffy (1981), vivimos en un mundo de sistemas en todos sus niveles: físico, químico, biológico, psicológico y sociocultural, es decir, que "todo está relacionado con todo" y, por ello, puede ser impredecible, violento, dramático y difícil de ser controlados solamente por las leyes de la probabilidad.

Este modelo exige la construcción de un metalenguaje, en el cual se puedan enunciar sin ambigüedades los términos de todas las disciplinas integrantes, lo que los enciclopedistas clásicos franceses (Diderot, d’Alambert, Condorcet y otros) iniciaron al tratar de albergar a "todo conocimiento digno de ser conocido" y lo que posteriormente Eco (2008), llamó "la búsqueda del lenguaje perfecto". Igualmente Focault (1978), en su obra Arqueología del saber, 1969, dirige su análisis hasta esta misma dirección, al buscar similitudes conceptuales en disciplinas tan disímiles como la economía, la lingüística y la biología, encontrando semejanzas en sus patrones de análisis y de cambio.

Por ello, el modelo transdisciplinar supone que, para alcanzar los resultados deseados, hay que tener presente lo que mencionan los lingüistas, como Ferdinand de Saussure (1931), al advertir que no existe nexo alguno entre el signo y su referente, esto es, que las palabras tienen un origen arbitrario o convencional; igualmente, se considera que es esencial superar las fronteras estructurales lingüísticas que separan una disciplina de otra y, simultáneamente, involucrarse en un diálogo intercultural.

Lógica polirrelacional. El método científico tradicional ha sido sustentado por la lógica lineal unidireccional, ya sea en una línea "deductiva" como en una "inductiva" La debilidad de la lógica inductiva está en el hecho de 
que los resultados están sujetos a un nivel de probabilidad de error aceptable entre el $1 \%$ y $5 \%$. Es evidente que la mayor debilidad de la lógica lineal es su irrealidad; es decir, su lejanía de la realidad concreta, especialmente si se trata de problemas de la ciencias humanas, donde no se da únicamente una variable independiente, una dependiente y una relación de causalidad, sino que siempre entran en juego muchas variables que no son lineales, ni unidireccionales, ni solamente causales, sino variables que interactúan mutuamente y entre las cuales se da toda clase y tipo de relaciones: de causa, condición, contexto, soporte, aval, secuencia, asociación, propiedad, contradicción, función, justificación, medio, etc.

A la luz de este análisis, el diálogo en la categoría de método, debe sustentarse en la lógica polirrelacional, que permita la comprensión e inteligibilidad de la realidad en general, y del comportamiento humano.

La lógica polivalente del diálogo como método presenta la siguiente dinámica: conocimiento, entendimiento y conocimiento resignificado, y se repite este ciclo dialéctico tanta veces se confrontan los participantes en forma proactiva y fehaciente. El entendimiento debe ser un debate abierto, con la posibilidad de divergencia. Aunque no debe necesariamente encaminarse a un debate divergente, es indudable que permita la exploración de muchas ideas y que se formen soluciones más completas y globales. Igualmente, los conocimientos renovados no se forman para subsistir permanentemente fijos, continúan cambiando y desarrollándose a medida que surgen nuevos puntos de vista o nuevos contextos.

\section{El diálogo como método}

\subsection{Características del diálogo como método}

Es una herramienta operativa de comunicación. Con el diálogo se intenta asimilar o, al menos, comprender los puntos de vista, las visualizaciones y el conocimiento de los otros. Lo que importa en el diálogo es el acceso a los significados de otros a nivel de estructuras profundas (ideas) y estructuras superficiales (símbolos) (Barnes, 1985). El diálogo como método es el encuentro de los hombres por encontrar la verdad de los hechos y de la vida.

Es un medio que lleva a un nuevo espacio intelectual. El hecho de comprender las ideas de los demás, nos interpela a desarrollar métodos, técnicas y estrategias en el hallazgo de un nuevo espacio intelectual y de una manera mental y vivencial compartida. 
Es un proceso incluyente. En la intensión de lograr una mayor comprensión de tipos o modelos de situaciones problemáticas, se requiere desarrollar un sentido de apropiación común del método dialógico y convertirse en parte interesada en la identificación de nuevos planteamientos para resolver estos tipos y otras variantes que se presentan en el entorno de aprendizaje como retos comunes.

Implica aprender y no solo conversar. El diálogo como método no implica solamente ser parte del grupo sino adoptar un nuevo estilo de hablar, pensar, y de comunicarse con los demás. Esto es, se requiere del autorreflexión, el espíritu de indagación y el cambio personal estén presentes. Se debe estar dispuesto a indagar las cuestiones profundas de los hechos y no solo los aspectos observables a primera vista.

Requiere de respeto mutuo. El que participa en el diálogo debe mostrar respeto y neutralidad por la opinión de los otros, identificar las diferencias, las coincidencias y demostrar capacidad para integrarlos en un nuevo conocimiento. El moderador del diálogo debe garantizar el respeto, afecto y la confianza de los participantes, que permita potenciar la voluntad de forjar nuevos conocimientos. De manera que puedan sentirse libres de expresar sus ideas sin miedo a las burlas o al rechazo.

Pone énfasis en una perspectiva temporal. Cómo herramienta metodológica de resolución de problemas, al evaluar el procedimiento de solución, surge el conflicto cognitivo de descubrir nuevas aplicaciones y elaborar nuevas formulaciones de los problemas modificando o agregando las condiciones iniciales. Esta postura de trabajo académico, que requiere tiempo y paciencia, puede ser rápido o extremadamente lento, abarcando años.

No es un método único de resolución. No es un método para resolver todo tipo de problema que surge en la ciencia. Más bien, el diálogo como método, representa un método dentro del conjunto de métodos en la comprensión y solución de problemas. El diálogo es flexible y aplicable a diferentes contextos de aprendizaje.

No hay ganadores. El objetivo del diálogo es integrar los diferentes puntos de vista o conocimientos en uno nuevo. Esto es compartir perspectivas, una y otra vez, y así descubrir nuevas ideas. Ninguna idea es totalmente desechada, todos aportan pero no hay ganadores. 


\subsection{Perfil de un líder facilitador}

Manejar las expectativas. Asegurarse que los objetivos y la definición de éxito queden planteados desde el inicio. Dejar bien claro que el diálogo no es la meta en sí mismo sino más bien un medio para alcanzar la meta.

Ser flexibles y adaptables al ritmo y estilo de aprendizaje. Ser sensible a los diferentes niveles de desarrollo cognitivo de los estudiantes y apoyarlos en su proceso de maduración.

Brindar un fuerte sentido de apropiación. El diálogo no debe ser percibido como algo impuesto por el docente. Esto permite que las voces se escuchen y que los grupos de aprendizaje se sientan más responsables de los resultados descubiertos. El docente debe levantar un "mapeo de participación" y estar atento en el involucramiento de los estudiantes. Para los apáticos, debe buscar las maneras de atraerlos a la interacción.

Gestionar el diálogo como método de descubrimiento. El docente debe motivar el desarrollo de la capacidad de visualización en la descomposición y composición mental de los elementos del problema, encontrar analogías y metáforas aplicables en la solución, a pensar con signos y símbolos en el planteamiento del problema, y a usar representaciones gráficas que faciliten la comprensión.

Planificar las sesiones de diálogo. Bosquejar las rutas lógicas generales por donde se conducirán los alumnos, elaborar las grandes preguntas en una variedad de formas equivalentes para la comprensión del problema. El docente tiene que prepararse continuamente.

\section{Sabiduría de los proverbios bíblicos en el diálogo como método en la solución de problemas}

En la mayoría de los libros de matemática, los problemas se formulan según los siguientes criterios: por afinidad de las operaciones que se requieren para su solución, por el nivel de complejidad, y por la claridad de la información que suministran. Todos estos criterios permiten establecer un conjunto de modelos o tipos de situaciones problemáticas para que el estudiante pueda poner a prueba su capacidad creativa en la solución. La experiencia de aprendizaje es gradual, el estudiante debe pasar por la siguiente gradación: 1) modelos que desarrollan confianza, pensamiento lógico, divergencia y 
flexibilidad, donde el número es lo de menos, 2) modelos que ayudan a estructurar la concordancia lógica entre las partes que componen un problema: datos, preguntas y los enunciados que lo relacionan, 3) modelos en donde existen una diversidad de enfoques y pluralidad de alternativas, 4) modelos que ayudan a ver el problema como un todo, donde hay un sistema de relaciones múltiples, y 5) modelos de extensión de ideas, donde prima la originalidad, imaginación y creatividad.

Frente a esta gran variedad de modelos, el profesor tiene una gran oportunidad para desarrollar el intelecto de sus alumnos si aplica el diálogo como método en la comprensión y solución de situaciones problemáticas. Si dedica su tiempo a ejercitarlos en procesos y operaciones rutinarias, matará en ellos el interés e impedirá su desarrollo intelectual. Si, por el contrario, a partir de un modelo graduado, pone a prueba la curiosidad activando las facultades inventivas y la aptitud del pensamiento reflexivo que, si son bien canalizadas, se puede experimentar el encanto del descubrimiento y el gozo de haberlo resuelto. Experiencias de esta modalidad de diálogo, vía preguntas generales y respuestas, puede determinar una afición para el trabajo intelectual e imprimirle una huella imperecedera en la mente y en el carácter.

Adecuando la postura de Polya (1990, p.18), para resolver un problema se necesita:

1. Comprender el problema en toda su magnitud.

2. Concebir un plan.

- Determinar la relación entre los datos y la incógnita.

- De no encontrarse una relación inmediata, puede considerar problemas auxiliares.

- Obtener finalmente un plan de solución.

3. Ejecutar el plan.

4. Examinar la forma de solución y los resultados obtenidos.

Cada uno de estos pasos es importante. Puede suceder que a un alumno se le ocurra una idea interesante y omitiendo todo el trabajo preparatorio, vaya directamente a la solución. Tales situaciones son deseables, naturalmente, pero se puede llegar a un resultado erróneo. Es de temerse lo peor si el alumno se lanza a realizar cálculos o trazos geométricos sin haber comprendido el problema. Es imposible ocuparse de los detalles si no se ha visto las relaciones esenciales. Se pueden evitar muchos errores si el alumno verifica cada paso al elaborar el plan. 
Ciertos proverbios bíblicos conservan la esencia misma de las observaciones que se requieren en el diálogo como método para la discusión de una situación problemática. Esto es, caracterizan de modo sorprendente los diversos procedimientos que se siguen al resolver un problema, las consideraciones de sentido común, los procedimientos y los errores clásicos. Se encuentra muchas observaciones perspicaces, claro está, unidas a un motivo determinado, expresado en el título del artículo, de una manera coherente y sin contradicciones.

Este artículo es un intento de reunir aquellos proverbios que grafican las etapas de la solución, siguiendo los cuatro pasos:

1. Comprender el problema. Lo que hace falta ante todo es comprender que: al que bien entiende, bien le va,... (Pr 16:20).

Los planes bien meditados dan buen resultado; lo que se hacen a la ligera causan la ruina ( $\operatorname{Pr} 21: 5)$. Tenemos que ver claramente los fines a los que tendemos: La sabiduría es la meta del inteligente, pero el necio no tiene meta fija ( $\operatorname{Pr}$ 17:24). Desgraciadamente no siempre se sigue y con frecuencia se reflexiona, se habla, se obra a tontas y a locas, sin haber comprendido realmente cuál es el propósito deseado: Es una necedad y una vergüenza responder antes de escuchar ( $\operatorname{Pr} 18: 13)$, un solo error causa grandes destrozos (Ec 9:18), las muchas prisas provocan errores ( $\operatorname{Pr} 19: 2)$. Si el fin perseguido no está claro en nuestra mente, perderemos fácilmente el camino y abandonaremos el problema.

No basta comprender el problema, hay que desear también solucionarlo. No podremos resolver un problema difícil sin un gran deseo de hacerlo; pero si tal deseo existe, concretaremos que es una honra investigar lo difícil (Pr 5:27).

2. Concebir un plan. Trazar un plan, concibiendo la idea de la acción apropiada, es lo esencial para llegar a la solución de un problema.

Una buena idea o una inspiración provienen de Dios. Pide con todas tus fuerzas, inteligencia y buen juicio; entrégate por completo a buscarlos, pues el Señor es quien da la sabiduría. ( $\operatorname{Pr} 2: 3,4$ y 6). La perseverancia y la paciencia conducen al éxito: Ser paciente es muestra de mucha inteligencia; ser impaciente es muestra de gran estupidez ( $\operatorname{Pr} 14: 29)$. Sin embargo, no basta repetir los intentos; debemos probar otros medios, cambiar de método. De todo esfuerzo se saca provecho ( $\operatorname{Pr} 14: 23)$. 
3. Ejecución del plan. Hay que poner el plan en ejecución en el momento oportuno, es decir, cuando ha madurado y no antes. No hay que hacerlo precipitadamente. El prudente se fija por dónde anda ( $\operatorname{Pr} 14: 15)$.

Debemos emplear nuestro juicio para elegir el momento propicio. He aquí una advertencia que subraya el error más común, el fracaso más corriente de nuestro juicio: Vale más lo que uno ve, que lo que se imagina (Ec 6:9). Nuestro plan, por lo general, no da solo una idea de conjunto. Tenemos que cuidar que los detalles encuadren en la línea general, para lo cual tenemos que examinar cuidadosamente cada detalle, uno tras otro: Cuando no hay consulta, los planes fracasan ( $\operatorname{Pr} 15: 22)$.

4. Examinar lo solución obtenida. Una etapa importante e instructiva del trabajo es volver a examinar la solución, una vez que se la ha obtenido. Los ríos van todos al mar,... y vuelven los ríos a su origen para recorrer el mismo camino (Ec 1:7).

Gracias a este nuevo examen podemos confirmar el resultado. Hay que hacer observar al principiante cuál es el tipo de confirmación que tiene valor: Al ver esto, lo grabé en mi mente; lo vi y aprendí esta lección ( $\operatorname{Pr} 24: 32)$ y el que aprende y pone en práctica lo aprendido, se estima a sí mismo y prospera ( $\operatorname{Pr} 19: 8)$.

Existen otros aspectos del desarrollo de la solución más sistemáticos y más elaborados, no sé si será posible que provengan de los proverbios bíblicos. Se recomienda que se investigue dicha posibilidad.

\section{Ejemplo con un tipo de problema de suma para primaria, categoría} combinación

Problema:

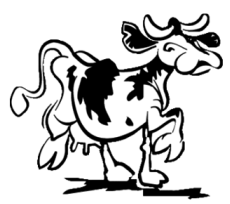

La primera vaca ha dado

La segunda vaca ha dado
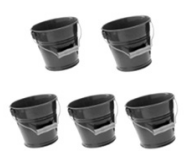

¿Cuántos baldes de leche han dado las dos vacas?

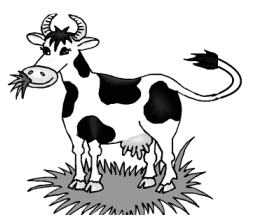

baldes de leche

baldes de leche 
Observación para el profesor: La reenunciación oral o escrita del problema es fundamental para su comprensión, ya que un niño(a) que no es capaz de explicitar la estructura del problema o, al menos, de representarlo gráficamente, difícilmente podemos decir que lo entienda.

Comprender el problema: Dibuja en el recuadro lo que nos dice el problema. Ten en cuenta Pr 16:20: Al que bien entiende, bien le va.

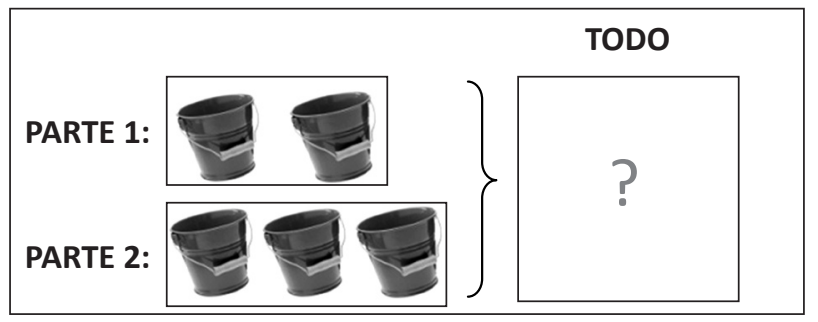

En tus propias palabras, puede reescribir el problema en función de lo que conoces (datos) y de lo que no se conoce (pregunta), partiendo de tu dibujo. Es una ayuda muy importante, al igual que el río recorre su mismo camino continuamente (Ec 1:7), reenunciar el problema, al menos, una vez.

Una formulación sería:

La primera vaca ha provisto dos baldes de leche, la segunda tres baldes de leche. Se quiere saber ¿Cuántos baldes de leche se tiene en total?

Concebir un plan: Esfuérzate, el Señor es quien da la sabiduría ( $\operatorname{Pr} 4: 6)$ Pinta los recuadros con tus respuestas

\begin{tabular}{|c|c|c|}
\hline ¿De qué trata el problema? & De reunir & De separar \\
\hline $\begin{array}{l}\text { ¿Qué sucede si se juntan } \\
\text { todos los baldes? }\end{array}$ & Más baldes & Menos baldes \\
\hline $\begin{array}{l}\text { ¿Qué operación tenemos } \\
\text { que hacer? }\end{array}$ & Sumar & Restar \\
\hline
\end{tabular}

Ejecución del plan: Fíjate por dónde vas (Pr 14:15), para luego hacer la cuenta Con el resultado que has logrado, contesta la pregunta del problema. 
¿Puedes hacer la cuenta?

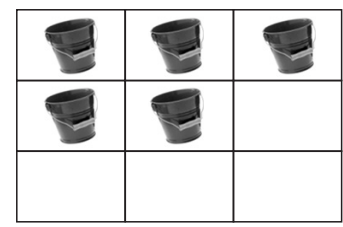

¿Cuánto baldes de leche tenemos?

5 baldes

Examinar el resultado obtenido: El que aprende y pone en práctica lo aprendido,..., prospera ( $\operatorname{Pr} 19: 8)$

¿Cómo has llegado a la solución?

Reuniendo las partes

para tener el todo

¿Acertaste antes de hacer la cuenta?
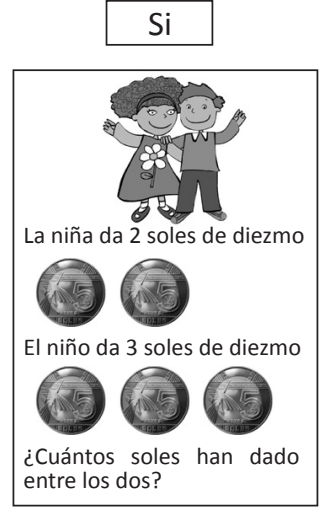

¿Puedes cambiar la naturaleza de los datos del problema para tener la misma solución?

¿Ahora, puedes cambiar los datos necesarios, del problema que has resuelto, para obtener una solución dada y distinta a la que obtuviste? 


\section{Conclusiones}

El escenario científico actual, caracterizado por la complejidad de los hechos, la transdisciplinariedad de la investigación y la emergente lógica polirrelacional, brinda los insumos conceptuales para el desarrollo del diálogo como método en la comprensión de modelos de situaciones problemáticas, en general de la realidad, y del comportamiento humano.

La lógica polirrelacional que sustenta al diálogo como método presenta la siguiente dinámica: conocimiento, entendimiento y conocimiento resignificado.

Valorar el conjunto de proverbios bíblicos citados en este artículo, pues proporcionan las grandes preguntas que guían los cuatro pasos en la solución de un problema.

Es importante precisar que la aplicación de los textos bíblicos tiene, por supuesto, implicaciones que van más allá del proceso sistémico de investigación.

Finalmente, cada uno de nosotros debemos procurar honrar a Dios incrementando nuestra fortaleza mental y espiritual e impartiendo continuamente las bendiciones concedidas. 


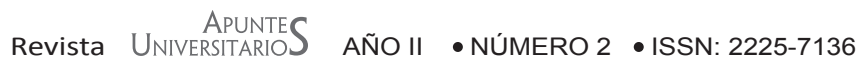

\section{Referencias}

Adorno, T. (1975). Dialéctica negativa. Madrid: Taurus.

Barnes, D. (1976). From Communication to Curriculum. Harmondswort, Penguin Books.

Bertalanffy, L. Von, y otros (1981). Tendencias en la teoría general de sistemas. Madrid: Alianza.

Eco, Umberto. (2008). La búsqueda de la lengua perfecta. Edición electrónica en: www. philosophia.cl / Escuela de. Filosofía Universidad ARCIS.

Foucault, M. (1978). La arqueología del saber. México: Siglo XXI.

Kant, I. (1973). Crítica de la razón pura. Buenos Aires: Losada.

Kuhn T.S. (1978). La estructura de las revoluciones científicas. México: FCE.

Martínez, M. (1996). Comportamiento humano: Nuevos métodos de investigación, 2da edic. México: Trillas.

Polya, George (1990). Cómo plantear y resolver problemas. México: Ed. Trillas.

Santa Biblia (1996). Versión Dios Habla Hoy. Sociedades Bíblicas Unidas.

Saussure, F. de (1931). Cours de Linguistique Générale. París: Payot. 